\title{
$3-530-4$
}

\section{Identification of pathological factors for Alzheimer disease using CRISPR/Cas9-based screening system}

\section{Taisuke Tomita}

Dept Neuropathol Neurosci, Grad Sch Pharm Sci, Univ Tokyo

Alzheimer disease $(\mathrm{AD})$ is pathologically characterized by the deposition of the amyloid- $\beta$ peptide $(\mathrm{A} \beta)$ as senile plaques in the brain. A $\beta$ is generated through the sequential cleavage of amyloid precursor protein (APP) by $\beta$-site APP cleaving enzyme 1 (BACE1) and $\gamma$-secretase. Whereas the aberrant metabolism of $\mathrm{A} \beta$ has been implicated in the etiology of $\mathrm{AD}$, the precise regulatory mechanism of $\mathrm{A} \beta$ generation remains unclear. In this study, we established a genetic screening based on the CRISPR/Cas 9 system to identify novel regulators of $\mathrm{A} \beta$ production. And we successfully identified calcium and integrin-binding protein 1 (CIB1) as a negative regulator of A $\beta$ production. CIB1 is a ubiquitously expressed, myristoylated, calcium-binding adaptor protein. We found that CIB1 interacts with the $\gamma$-secretase complex. Moreover, the disruption of Cib1 specifically reduced the cell-surface localization of the $\gamma$ secretase complex, without changing the intrinsic activity of $\gamma$-secretase. Finally, we confirmed using the single-cell RNA-seq data in human that CIB1 mRNA level in neuron was decreased in the early stage of AD. Taken together, our results indicate that CIB1 regulates $\mathrm{A} \beta$ production via controlling the subcellular localization of $\gamma$-secretase, suggesting $\mathrm{CIB} 1$ is involved in the development of $\mathrm{AD}$. 